\title{
Image Compression using HAAR Wavelet Transform and Discrete Cosine Transform
}

\author{
Khushpreet Kaur \\ Master of Technology in CSE, SGGSWU, \\ Fatehgarh Sahib, \\ Punjab, India
}

\author{
Sheenam Malhotra \\ Assistant Professor, Department Of CSE, \\ SGGSWU, Fatehgarh Sahib, \\ Punjab, India
}

\begin{abstract}
The main objective of the method is to provide the interesting part of the medical image on which different treatements are to be implemented. In medical image when some portion of image is to be selected, then ROI is selected with the help of Discrete Cosine Transform. It is used with the ROI technique to compress the medical image to remove the blocking effect. The different filters will be applied to the images. The aim of this method is to analyze the results using MATLAB software and calculate various parameters such as CR, entropy, bits per pixel, PSNR, MSE, to compress the ROI part of the color images without losing any contents of the images and to maintain the storage memory space. Imaging helps a lot to represent the internal problem of body in visual manner. Various medical diagnosing techniques are using digital images of human body as the deciding factors for next medical treatment. The new techniques are enhanced to compress the medical image so that the problems encountered in the previous study can be solved.
\end{abstract}

\section{Keywords}

Entropy, PSNR, MSE, Haar Wavelet Transform, Discrete Cosine Transform, Region of Interest.

\section{INTRODUCTION}

Data compression uses different calculation methods and mainly be divided into two categories. According to the distortion of image data, they are lossless compression and lossy compression. Lossless compression refers to the data that during the compression process won't be lost yet and can restore to its pre-compression status. Things are diametrically opposed to the lossy compression [3]. Compression is achieved by the removal of redundancies. The image is converted from analog to digital form for processing, storage and transmission by computer applications.

Image compression is also used in the Medical imaging. Medical imaging helps a lot to represent the internal problem of body in visual manner. The ROI part is also selected in the medical imaging because the general theme is to preserve the quality in diagnostically critical regions, but allowing

lossy compression of the other regions.

Some of the most desirable properties of any compression method for the medical images include:

- High lossless compression ratios

- Resolution scalability, which refers to the ability to decode the compressed image data at various resolutions and

- Quality scalability, which refers to the ability to decode the compressed image at various qualities or signal-to-noise ratios (SNR) up to lossless reconstruction [1].
Image compression plays a key role in many important applications, including image database, image communications, remote sensing, the use of satellite imagery for weather and other earth-resource application [4]. In image compression reducing the storage requirement is equivalent to increasing the capacity of the storage medium and hence communication bandwidth. Thus the development of efficient compression techniques will continue to be a design challenge for future communication systems and advanced multimedia applications [5]. The compressed image is decompressed to reconstruct the image and an approximation of it. So image compression is used to minimize the amount of memory needed to represent an image [7].

\section{ROI BASED COMPRESSION TECHNIQUES}

There are a number of methods for compressing medical images. Every method has their own advantages and disadvantages. Some of the major algorithms being used are as following:

SPIHT Method SPHIT method is powerfull, efficient and computationally simple image compression algorithm [6].The input images are segmented into the foreground and background respectively and a chain code based shape coding scheme is used to code the ROI's shape information. Then, the critically sampled shape adaptive integer wavelet transforms (IWTs) are performed on the foreground and background image separately to facilitate lossy to lossless coding. Finally, the shape-coding bit stream, the foreground bit stream, and the background bit stream are combined into a single bit stream. For SPIHT algorithm it can be seen that the compression ratio increase, when the levels of decomposition is increased [8]. This is because, when the levels of decomposition are increased, coefficients with higher magnitude concentrate mostly on the root levels. Also most of the coefficients will have low magnitudes. These coefficients require only less number of bits to be transmitted. Hence the compression ratio will increase when decomposition level is increased. Encoding and decoding process occur at a faster rate when SPIHT is combined with discrete wavelet transform [9].

Discrete Cosine Transform Discrete Cosine Transform (DCT) is used to achieve high compression ratio without degrading of quality. The DCT helps to separate the image into parts (or spectral sub-bands) of differing importance (with respect to the image's visual quality) [2]. The DCT is similar to the discrete Fourier transform: it transforms a signal or image from the spatial domain to the frequency domain. DCTs are important to numerous applications in science and engineering, from lossy compression of audio (e.g. MP3) and images (e.g. JPEG), where small high-frequency components 
can be discarded, to spectral for the numerical solution of partial differential equations.

The order of values obtained by applying the DCT is coincidentally from lowest to highest frequency. When reconstructing the data and transforming it back to the spatial domain, the results are remarkably similar to the original signal. The DCT method can be used to compress both color and gray scale images . DCT is a method most frequently used in several areas including WWW, industries, science and engineering etc.

Adaptive Arithmetic entropy Coding Arithmetic coding is different from other coding methods for which we know the exact relationship between the coded symbols and the actual bits that are written to it. It codes one data symbol at a time and assigns to each symbol a real-valued number of bits [9]. The main feature of this algorithm includes two schemes: the method of computing approximate distorted area and the method of adaptive sampling.

HAAR Wavelet Transform The haar transform (HT) is one of the simplest and basic transformations from a space domain and a local frequency domain. This method reduces the calculation work. HT decomposes each signal into two components. One component is called average and other is known as difference [6]. It is used to reduce the memory requirements and the amount of inefficient movement of Haar coefficients. The drawback in the number of addition and subtraction operation can be balanced by decreasing the number of division operations, especially when used at low bit rates,this introduces compression artefacts.

\section{IMPLEMENTATION WORK}

The method will uses the ROI part with the Discrete Cosine Transformation. It is a major challenge to compress the medical image to reduce the storage space, bandwidth utilization, blocking effect and other disadvantages. So there is a need to modify the existing techniques to get the advantage of these methods. DCT will uses with the ROI technique to compress the medical image to remove the blocking effect.

\subsection{Research plan}

The fig. 1 is a flow diagram of medical image which shows the steps that are performed while compressing an image using ROI method. In it first the medical image is segmented into parts and then ROI and non-ROI parts of the image are defined, based on which the compression is done. Then the image is transmitted to the receiver where the decompression process is carried out to check the quality of the image with ROI.

Step 1: Medical image is input for the process which may be ant type of medical image which have to process (MRI/CT/DICOM).

Step 2: The image is segmented into different parts.

Step 3: The classification of image is described.

Step 4: Describes that whether the image is selected for ROI or other than ROI.

Step 5: If the image is selected with ROI then it is compressed with lossless compression otherwise lossy compression is used.

Step 6: Compression of image is done.

Step 7: Image is transmitted to the desired system.

Step 8: Image is received at receiver side.

Step 9: Image decompression is done.

Step 10: Correct image is received with lossless ROI compression.

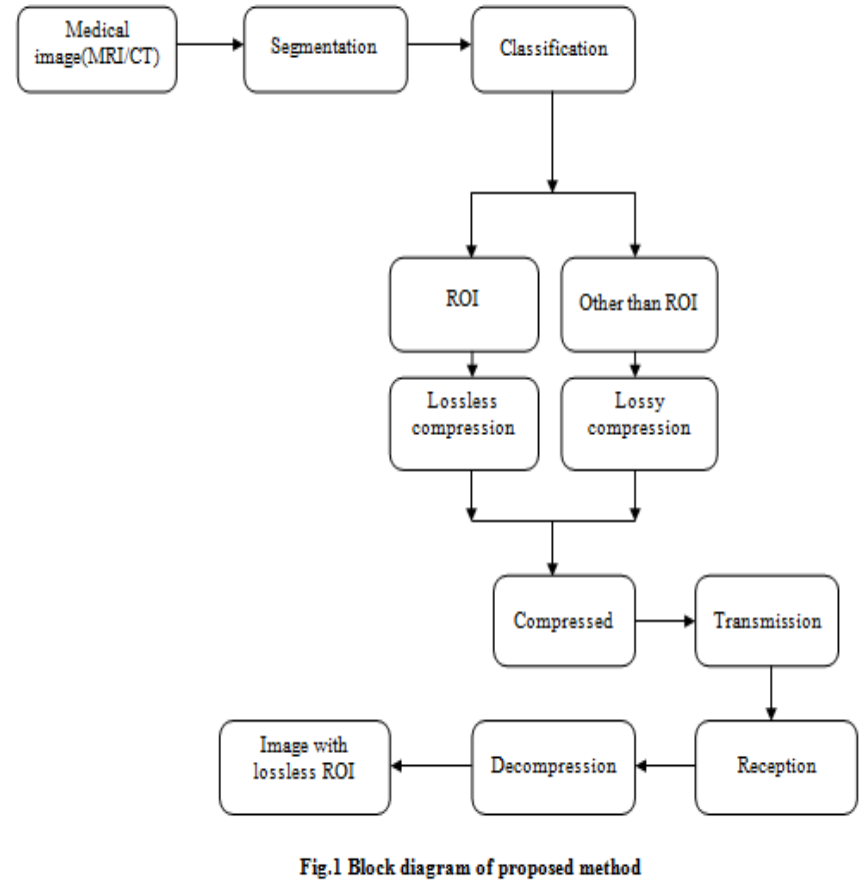

\subsection{ROI Selection}

Region of Interest is important in medical applications where certain parts of the image are of higher diagnostic significance than others. Fig. 2 demonstrates the implementation design of selection of the ROI part. The image is first selected from the image folder and then the selection of the interested or required portion is done. The cursor that is moving on the screen is used to select the image ROI part. The interested part or the part of medical image where the disease will occurs is selected with the help of the movement of cursor. The part that is selected from the medical image is the part that the experts will select to test or to read the effected part of the image. The cursor can be moved in any of the four directions from where we have to select the interested part.

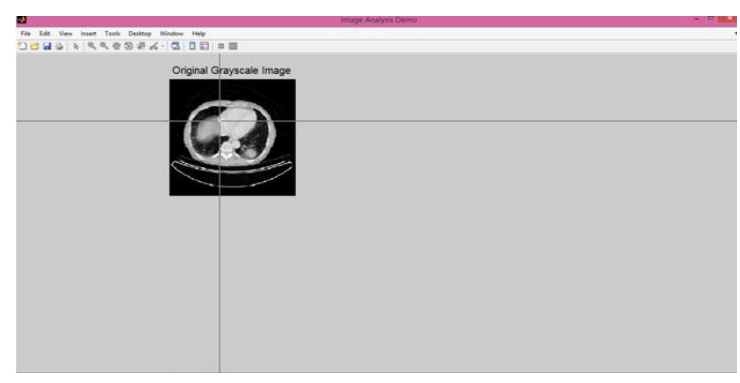

\subsection{Output of ROI}

The output of ROI is the selected part of the medical image. Fig. 3 describes that the image that is selected with the help of the moving cursor is displayed in this part of the image, is ROI image. The output will shows the original image and along with the original image it will shows the output of the ROI part which is region of interest that we have selected for the implementation. 


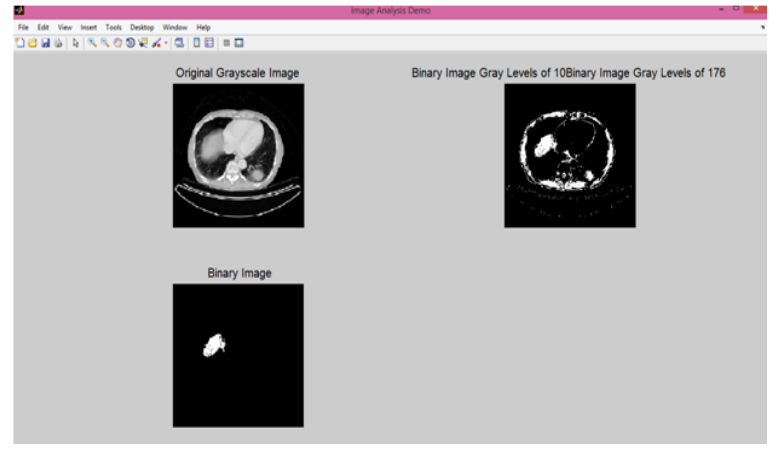

Fig.3 Output of the ROI part of image

\subsection{Haar Wavelet image processing}

The Haar wavelet can be used on both ROI and Non-ROI parts of the image. Fig. 4 describes the output of processing of the image with the help of Haar Wavelet Transform method, which shows the output in the form of total compression and gain compression. The output of the image is shown in the form of peak signal to noise ratio and mean square error. The input image entropy and the output image entropy is also shown when we compress or decompress the image with the help of Haar Wavelet method. With the increase in PSNR value, the image quality will also be increased and image quality will be decreased if the value of MSE will increased.

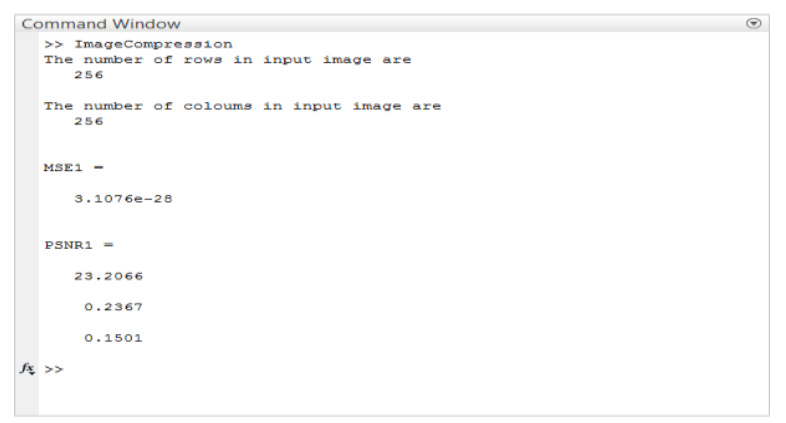

Fig.4 Haar Wavelet image processing

\subsection{Reconstruction of image with Haar wavelet transform}

In the reconstruction of image the Fig. 5 describes about the compression and decompression of the image. The image that we have compressed is checked for the correction with the help of image decompression. When the image is compressed and decompressed with the help of Haar Wavelet, then after decompression the error will occurs in the image. The error that will occur in the image is shown in the form of mean square error and peak signal to noise ratio. The error will be occurred in the image when the reconstructed image is not equal to the original image. To construct the image with no error we will compress and decompress the image with the help of a special method discrete cosine transformation. The image quality will be described in the form of mean square error, if the error is less in the image then the image quality will be good. So the main focus is to reduce the errors and the blocking effect that has occurred in the image. In IWT the image processing will be done on all the image which is in the form of horizontal, vertical and diagonal processing of the image.

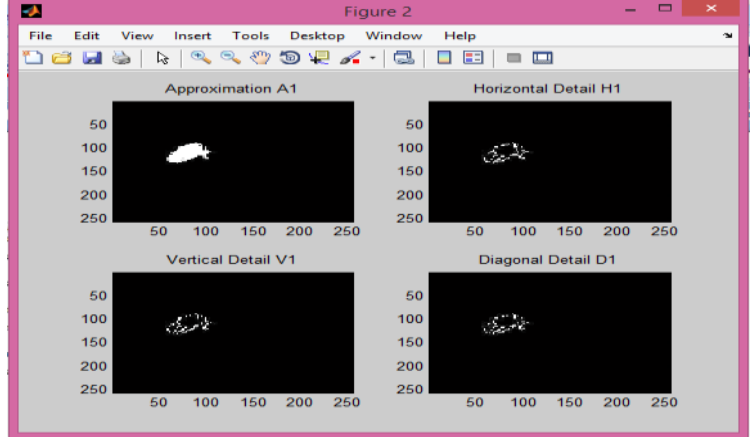

Fig.5 Reconstructed image with blocking effect in Haar Wavelet method

F. DCT and Haar wavelet output with parameters

The Table.1 describes about the DCT and Haar Wavelet output with different parameters like peak signal to noise ratio (db), mean square error, input image entropy (pixels), output image entropy (pixels) etc. These different parameters are used to compare the output or quality of the selected part of the image. The different graphs will be drawn on the basis of these parameters so that we can view the output with the help of graphs, which is easily understandable. Different type of images will be taken to compare the quality e.g. .bmp, .jpg, .png etc.

Table.1 DCT and Haar Wavelet output with different parameters

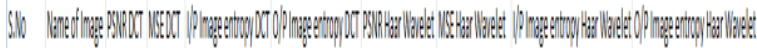

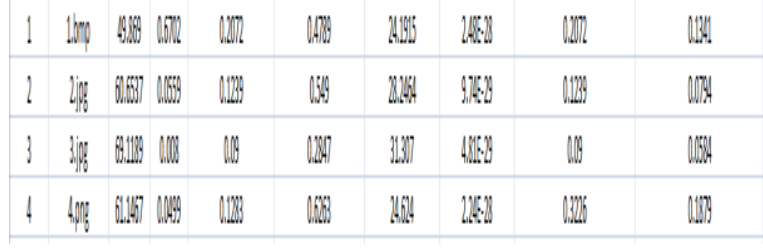

\subsection{Graphical representation of output values}

In this graphical representation the Fig. 6 describes about the graphical representation of the output values of the DCT and Haar Wavelet methods. The graph will shows that the output values of the DCT method are much better than that of Haar Wavelet method and also the image quality is high in case of discrete cosine transform. The DCT have higher values than other methods that is shown through the values that are given in the graph. The graph will shows the 4 series with different colors that are given for different values. The first point of all the series will gives the output of the first column and so on. The graph that has high values will have high quality of the selected point. The graph with the higher value will have high quality of the compressed and decompressed images.
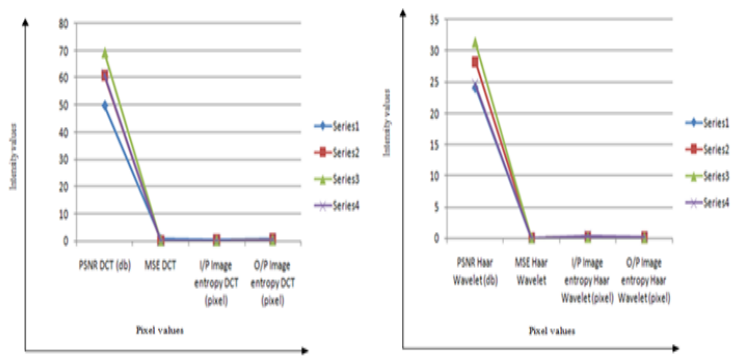

Fig.6 Graphical representation of output values 


\subsection{PSNR value representation}

The Fig.7 describes the PSNR value representation in the DCT and Haar Wavelet values. The blue line will gives the output of the DWT values which is more than that of the Haar Wavelet values and the red line will gives the output of the Haar Wavelet values which are less than that of DCT values. So the image quality of the image is better than the other cases in the form of DCT. The twist in the lines will describes the change in values in the images with the different formats. The image that have higher peak signal to noise ratio is the high quality image and the image with low values is low quality image. The graph will shows us the graphical representation of the values so that we can easily understand the values and the view of the quality of the image. Here the different type of images are taken and there values are compared on the basis of their output.

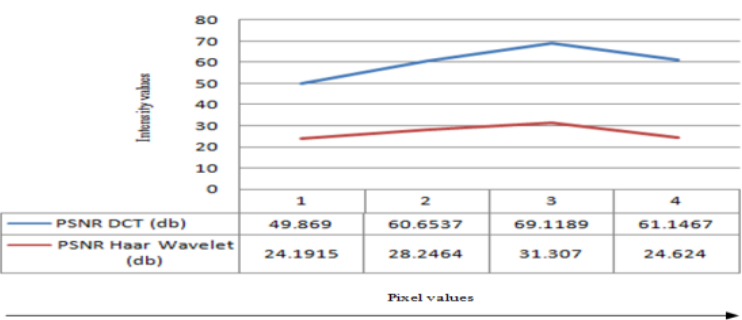

Fig.7 PSNR value representation

\subsection{Output image entropy representation}

The output image entropy is change in entropy value of the image. Fig.8 describes about the output image entropy representation of the different images to compare the quality of the image. The blue line will shows the o/p image entropy of the DCT values and the red line will shows the o/p image entropy of the Haar Wavelet values. The image with high o/p image entropy will have the high quality than others.

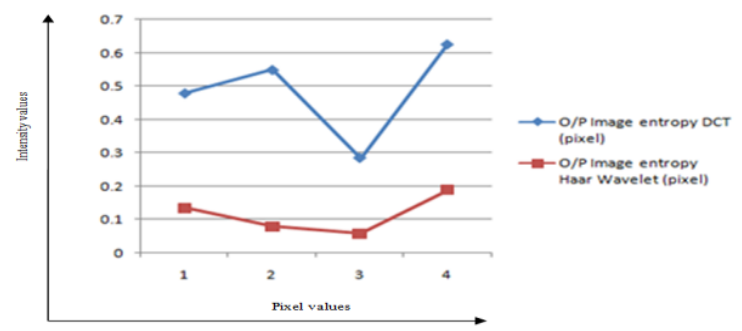

Fig.8 Output image entropy representation

\section{CONCLUSION AND FUTURE SCOPE}

Medical imaging has a great impact on the diagnosis of disease and surgical planning. The imaging devices continue to generate more data per patient, often large imaging. These data need long term storage and efficient transmission so there is a need to compress medical images. The various methods are used to compress the images. In all the research papers we have studied the various techniques, these techniques have several pros and cons. These are due to the methods that are used to compress the medical images. Imaging helps a lot to represent the internal problem of body in visual manner. Various medical diagnosing techniques are using digital images of human body as the deciding factors for next medical treatment. The new techniques are enhanced to compress the medical image so that the problems encountered in the previous study can be solved.

As all know that the usage of images in medical science is increasing day by day. It is a major challenge to compress the medical image to reduce the storage space, bandwidth utilization, blocking effect and other disadvantages. So there is a need to modify the existing techniques to get the advantage of these methods. In the future work the image compression is performed on the medical color image with the help of different techniques and morphological operator to get the better results in the future.

\section{REFERENCES}

[1] V.K. Bairagi, A.M. Sapkal "Automated region based hybrid compression for digital imaging and communications in medicine magnetic resonance imaging images for telemedicine applications," published in IET Science, Measurement and Technology, ISSN: 1751-8822,Issue No.4, Vol No.6, Page No. 247-253, 2012.

[2] Mr. Amit S. Tajne, Prof. Pravin S. Kulkarni "A Survey on Medical Image Compression Using Hybrid Technique," International Journal of Computer Science and Mobile Computing (IJCSMC), ISSN: 2320-088X, Issue No.2, Vol.4, Page No.18 - 23, Feb 2015.

[3] Li Zhiqiang, Sun Xiaoxin, Du Changbin, Ding Qun "JPEG algorithm analysis and application in image compression encryption of digital chaos," Third International Conference on Instrumentation, Measurement, Computer, Communication and Control, Page No. 185-189, 2013.

[4] Jagadish H. Pujar, Lohit M. Kadlaskar "A new lossless method of image compression and decompression using huffman coding techniques," Journal of Theoretical and Applied Information Technology, www.jatit.org, Page No.18-22.

[5] Maneesha Gupta, Dr.Amit Kumar Garg "Analysis Of Image Compression Algorithm Using DCT," International Journal of Engineering Research and Applications (IJERA), ISSN No. 2248-9622, Issue No.1, Vol.2, Page No. 515-521, Jan-Feb 2012.

[6] Navjot Kaur, Preeti Singh "A New Method of Image Compression Using Improved SPIHT and MFHWT," International Journal of Latest Research in Science and Technology, ISSN:2278-5299, Issue No.2, Vol.1, Page No.124-126, July-Aug (2012).

[7] Dalvir Kaur, Kamaljit Kaur "Huffman Based LZW Lossless Image Compression Using Retinex Algorithm,' International Journal of Advanced Research in Computer and Communication Engineering, ISSN: 2319-5940, Issue No.8, Vol. 2, Page No. 3145-3151, Aug 2013

[8] Lavanya. M, M. Suresh Kumar "intelligent compression of medical images Based on multi ROI," International Journal of Emerging Technology and Advanced Engineering, ISSN: 2250-2459, Issue No.1, Vol. 3, Page No. 490- 493, Jan 2013.

[9] Yung-Gi Wu "Medical Image Compression by Sampling DCT Coefficients," IEEE transactions on information technology in biomedicine, Issue No.1, Vol. 6, Page No.86-94, March 2002. 